\title{
Fluid and solid phases of the Gaussian core model
}

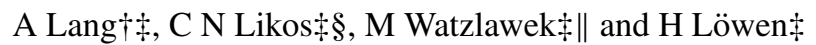 \\ $\dagger$ Institut für Theoretische Physik and CMS, Technische Universität Wien, \\ Wiedner Hauptstraße 8-10, A-1040 Wien, Austria \\ \$ Institut für Theoretische Physik II, Heinrich-Heine-Universität Düsseldorf, Universitätsstraße 1, \\ D-40225 Düsseldorf, Germany \\ E-mail: likos@thphy.uni-duesseldorf.de
}

Received 3 April 2000

\begin{abstract}
We study the structural and thermodynamic properties of a model of point particles interacting by means of a Gaussian pair potential first introduced by Stillinger (Stillinger F H 1976 J. Chem. Phys. 65 3968). By employing integral equation theories for the fluid state and comparing with Monte Carlo simulation results, we establish the limits of applicability of various common closures and examine the dependence of the correlation functions of the liquid on the density and temperature. We employ a simple, mean-field theory for the high-density domain of the liquid and demonstrate that at infinite density the mean-field theory is exact and that the system reduces to an 'infinite-density ideal gas', where all correlations vanish and where the hypernetted-chain (HNC) closure becomes exact. By employing an Einstein model for the solid phases, we subsequently calculate quantitatively the phase diagram of the model and find that the system possesses two solid phases, face-centred cubic and body-centred cubic, and also displays re-entrant melting into a liquid at high densities. Moreover, the system remains fluid at all densities when the temperature exceeds $1 \%$ of the strength of the interactions.
\end{abstract}

\section{Introduction}

The structural and phase behaviour of systems whose constituent particles interact by means of pair potentials diverging at zero separation between them is a problem that has been extensively studied in the last three decades, by a variety of theoretical, experimental and computational methods. It has become established that the diverging repulsions at close separations between the particles are the dominant factor causing crystallization of the system; indeed, for the purposes of understanding the freezing mechanism, the simple, hard-sphere model is in most cases sufficient. The crystal structure into which a system freezes depends on the steepness of the repulsion, with hard repulsions favouring a face-centred cubic (fcc) lattice and soft ones a body-centred cubic (bcc) lattice [1]. On the other hand, interparticle attractions are responsible for bringing about a liquid-gas coexistence, whose stability with respect to the freezing transition depends crucially on their relative range with respect to that of their repulsive counterparts [2].

Though repulsions are necessary to bring about a solidification transition, they are by no means sufficient. This was demonstrated recently by Watzlawek et al $[3,4]$ who studied a system interacting by means of an ultrasoft, logarithmically diverging potential which has

$\S$ Author to whom any correspondence should be addressed.

\| Present address: Bayer AG, Central Research Division, D-51368 Leverkusen, Germany.

0953-8984/00/245087+22\$30.00 @ C 2000 IOP Publishing Ltd 
been shown to model accurately the effective interaction between star polymers in a good solvent $[5,6]$. In particular, the pair potential employed in references $[3,4]$ reads as

$\beta v(r)= \begin{cases}\frac{5}{18} f^{3 / 2}\left[-\ln \left(\frac{r}{\sigma}\right)+\frac{1}{1+\sqrt{f} / 2}\right] & \text { for } r \leqslant \sigma \\ \frac{5}{18} f^{3 / 2} \frac{1}{1+\sqrt{f} / 2}\left(\frac{\sigma}{r}\right) \exp \left[-\frac{\sqrt{f}(r-\sigma)}{2 \sigma}\right] & \text { for } r \geqslant \sigma\end{cases}$

where $\beta \equiv\left(k_{B} T\right)^{-1}$ is the inverse temperature ( $k_{B}$ is Boltzmann's constant), $f$ is the functionality of the stars and $\sigma$ is the typical extent of the stars, the so-called corona diameter. It was found that the strength of the repulsion, controlled by the parameter $f$ in equation (1), is crucial in determining whether the system crystallizes. For values $f \leqslant 34$, the system remains fluid at all densities and, even for values $f>34$, re-entrant melting into a high-density liquid has been observed for a particular range of functionalities [3,4].

We are, therefore, faced with a question which is specular to the old question of how much attraction is needed in order to make a liquid [7], namely: How much repulsion is needed in order to make a solid? In this respect, it is interesting to consider the extreme family of potentials that are bounded, i.e., they remain finite for the whole range of interparticle separations, even at full overlap between the particles. In the context of microscopic interactions in atomic systems, such bounded potentials are evidently unphysical: full overlaps between atoms, molecules or even compact macromolecular entities are forbidden by the Born repulsions between the electrons. Yet, they are perfectly realistic in the context of effective interactions between fractal objects such as polymer chains, as will be demonstrated in section 2.

In comparison with the case for systems interacting by means of unbounded potentials, very little is known about bounded interactions. A model system that has been recently studied is the 'penetrable spheres model' (PSM), in which the interaction is a positive, finite constant for separations smaller than a diameter $\sigma$ and vanishing otherwise [8]. The model was studied by means of cell-model calculations and computer simulations [8], liquid-state integral equation theories [9] and density functional theory [10] and it was found that no re-entrant melting takes place with increasing density because a clustering mechanism stabilizes the solid at all temperatures. The PSM is, however, rather peculiar in this respect and both the constant value of the potential inside $\sigma$ and its sharp fall to zero outside $\sigma$ render it a rather unrealistic model for effective interactions.

A much more realistic model of a bounded potential is the Gaussian core model (GCM), introduced in the mid-1970s by Stillinger [11]. In the GCM, the interaction potential between the particles reads as

$$
v(r)=\varepsilon \mathrm{e}^{-(r / \sigma)^{2}}
$$

where $\varepsilon>0$ is an energy and $\sigma$ a length scale. Stillinger's original work [11] was complemented later by molecular dynamics simulations [12,13], high-temperature expansions [14] and the discovery of exact duality relations in the crystalline state [15]. On the basis of these considerations, a semi-quantitative phase diagram of the GCM was drawn $[11,16]$, which displayed re-entrant melting and a maximum freezing temperature $t_{\mathrm{u}}$. The latter is defined as the temperature above which the system remains fluid at all densities. Yet, a detailed study of the structure of the GCM fluid by means of the modern techniques of liquid-state theory and a quantitative calculation of the phase diagram of the GCM are still lacking. The purpose of this work is to fill this gap and to draw from the study of the GCM some general conclusions, applicable to a large class of bounded potentials. 
The rest of the paper is organized as follows. In section 2 we briefly review previous work on this model and make contact with effective interactions between polymer chains. In section 3 we present results concerning the structure of the fluid, obtained by means of liquid-state integral equation theories and Monte Carlo simulations. In section 4 we discuss the high-density limit of the fluid, by employing a simple mean-field density functional. In section 5 we present the theory for the crystal phases and in section 6 we make use of the results for the fluid and the solids in order to quantitatively draw the phase diagram of the system. Finally, in section 7 we summarize and conclude.

\section{Gaussian effective interactions}

A Gaussian pair potential was first proposed fifty years ago by Flory and Krigbaum [17], as the effective interaction between the centres of mass of two polymer chains. This so-called Flory-Krigbaum potential, $v_{\mathrm{FK}}(r)$, reads as

$$
\beta v_{\mathrm{FK}}(r)=N^{2} \frac{v_{\mathrm{seg}}^{2}}{v_{\mathrm{solv}}}\left(\frac{3}{4 \pi R_{g}^{2}}\right)^{3 / 2}(1-2 \chi) \exp \left(-\frac{3}{4} \frac{r^{2}}{R_{g}^{2}}\right)
$$

where $v_{\text {seg }}$ and $v_{\text {solv }}$ denote the volumes of a monomer segment and a solvent molecule, respectively, $N$ is the degree of polymerization, $R_{g}$ the radius of gyration of the chains and $\chi$ is a parameter that controls the solvent quality $(0<\chi<1 / 2$ denotes a good solvent and $\chi>1 / 2$ a poor one.)

Formally, the effective interaction potential $v_{\text {eff }}(r)$ between two polymer chains is defined as follows: the centres of mass of the two chains are held fixed at separation $r$, and the canonical trace is taken over all the monomer degrees of freedom. In this way, a restricted partition function $Q(r)$ is obtained, where the restriction denotes the constraint of holding the centres of mass fixed. The effective interaction is then simply the free energy $-k_{B} T \ln Q(r)$. As can be seen in figure 1, the centres of mass of two polymer chains can coincide without any violation of the excluded-volume condition for the monomers (beads). Hence, the effective interaction between polymer centres of mass does not have to diverge at $r=0$ and a bounded potential is fully realistic.

The effective interaction between linear polymers has been extensively studied by computer simulations, both on-lattice [18,19] and off-lattice [20-24] and for a varying solvent quality. In addition, Krüger et al [23] performed a theoretical calculation of the effective interaction for athermal solvents, using renormalization-group techniques. Regardless of the detailed manner in which the monomer-monomer excluded-volume interactions have been modelled, all these studies converge to the result that the effective potential $v_{\text {eff }}(r)$ is extremely well approximated by a universal Gaussian function $\dagger$ given by equation (2), with $\varepsilon$ being an energy scale of the order of the thermal energy $k_{B} T$ and $\sigma$ a length scale of the order of the spatial extent of the chains (e.g., the radius of gyration). This type of effective Gaussian interaction has been recently employed by Louis et al [25] in their study of the structure of colloid-polymer mixtures.

The Gaussian effective potential between polymer chains in purely entropic in nature, causing the energy $\varepsilon$ to scale with temperature. Therefore, the latter completely drops out of the problem as an irrelevant thermodynamic parameter. However, since the system is of significant theoretical interest, it is pertinent to consider $\varepsilon$ and $k_{B} T$ in more generality, as two

$\dagger$ The universality holds when the two chains have the same degree of polymerization and the solvent is athermal. If the temperature becomes relevant or the two chains have different numbers of monomers, corrections to the Gaussian form appear. 


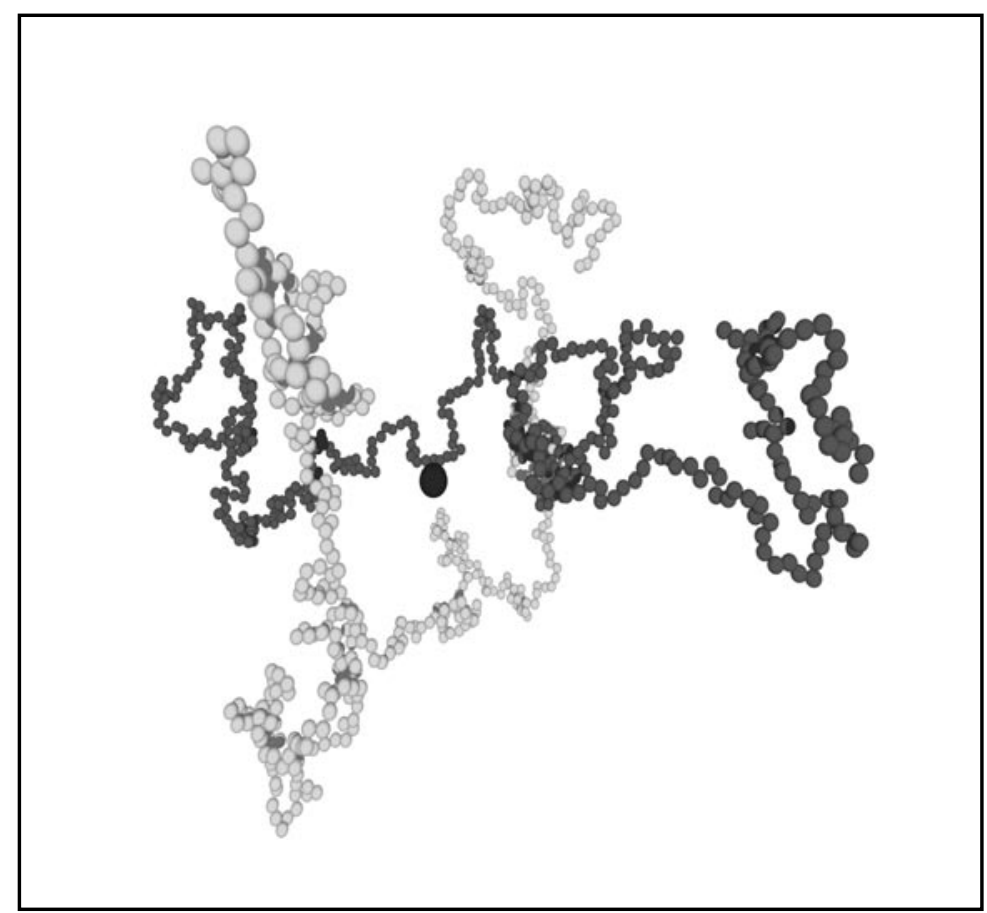

Figure 1. A snapshot from a simulation involving two self-avoiding polymers. In this configuration, the centres of mass of the two chains (denoted by the big sphere) coincide, without violation of the excluded-volume conditions. (Courtesy of Arben Jusufi.)

independent energy scales, and study the properties of the system as functions of density and temperature. To this end, we define the dimensionless temperature $t$ as

$$
t \equiv \frac{k_{B} T}{\varepsilon}=(\beta \varepsilon)^{-1}
$$

The density $\rho$ of a system of $N$ particles (or polymer chains in the above discussion) enclosed in the macroscopic volume $V$ is the ratio $N / V$. As dimensionless measures of the density, we are going to use to use in this paper both the parameter $\bar{\rho} \equiv \rho \sigma^{3}$ and the 'packing fraction' $\eta$, defined as

$$
\eta \equiv \frac{\pi}{6} \rho \sigma^{3}
$$

A number of exact properties for the GCM were worked out by Stillinger et al $[11,15,16]$, yielding important information on the topology of the phase diagram. The relevant properties are listed below.

(i) The hard-sphere limit. At low temperatures and densities, the GCM reduces to a hardsphere (HS) system with a HS diameter that diverges as the temperature approaches zero [11]. Hence, the GCM displays there the usual HS freezing transition from a fluid into a fcc lattice and the coexistence densities converge to zero at vanishing temperature. Using the known results for the HS freezing [26-28], the shapes of the freezing and melting lines $t_{\mathrm{f}}(\bar{\rho})$ and $t_{\mathrm{m}}(\bar{\rho})$ as $t \rightarrow 0$ are given by the equations

$$
t_{\mathrm{f}}(\bar{\rho})=\frac{1}{\ln 2} \exp \left(-\frac{0.962}{\bar{\rho}^{2 / 3}}\right)
$$




$$
t_{\mathrm{m}}(\bar{\rho})=\frac{1}{\ln 2} \exp \left(-\frac{1.027}{\bar{\rho}^{2 / 3}}\right) .
$$

(ii) Duality relations. Due to the property of the Gaussian potential of remaining form invariant under Fourier transformation, it has been shown that the internal energy of a certain crystal at low densities (lattice sum) is related to that of the reciprocal crystal at high densities [15]. In this way it was established that the fcc and bcc lattices are degenerate at $t=0$ at the 'magic density' $\bar{\rho}_{*}=\pi^{-3 / 2} \cong 0.1796$, with the fcc 'winning' for $\bar{\rho}<\bar{\rho}_{*}$ and the bec for $\bar{\rho}>\bar{\rho}_{*}$.

(iii) Re-entrant melting. At small but finite temperatures and at high densities, the bcc lattice remelts into a fluid and the high-density freezing and melting lines are given by the equations [15]

$$
\begin{aligned}
& t_{\mathrm{f}}(\bar{\rho}) \propto \exp \left(-K_{\mathrm{f}} \bar{\rho}^{2 / 3}\right) \\
& t_{\mathrm{m}}(\bar{\rho}) \propto \exp \left(-K_{\mathrm{m}} \bar{\rho}^{2 / 3}\right)
\end{aligned}
$$

with the appropriate constants $K_{\mathrm{f}}$ and $K_{\mathrm{m}}$.

Note the duality between equations (6), (7), valid for $\bar{\rho} \rightarrow 0$, and (8), (9), valid for $\bar{\rho} \rightarrow \infty$. Apart from proportionality constants, one pair can be obtained from the other by the formal substitution $\bar{\rho} \rightarrow 1 / \bar{\rho}$. Moreover, equations (8) and (9) show that the slopes of the high-density freezing and melting lines are negative. Contrary to the usual case, the liquid coexisting with the solid has a higher density than the latter.

The above general considerations were combined with molecular dynamics simulations $[12,13]$ and an approximate phase diagram of the model was drawn [16]. It was found that below an 'upper freezing temperature' $t_{\mathrm{u}} \approx 0.008$, the GCM displays the transitions fluid $\rightarrow$ $\mathrm{fcc}, \mathrm{fcc} \rightarrow \mathrm{bcc}$ and bcc $\rightarrow$ fluid with increasing density, whereas above $t_{\mathrm{u}}$ a single fluid phase exists and no freezing takes place, at any density. However, the liquid structure of the GCM has not been studied to date and the phase diagram was drawn only semi-quantitatively. In the following sections we perform a quantitative analysis on all these aspects, using standard tools of statistical mechanics.

\section{The fluid: integral equation theories and Monte Carlo simulations}

The theoretical determination of the pair structure of a uniform fluid amounts to the calculation of the radial distribution function $g(r)$ and the direct correlation function (dcf) $c(r)$ [29]. The correlation function $h(r)$ is simply $g(r)-1$ and then $h(r)$ and $c(r)$ are connected to each other through the Ornstein-Zernike relation which has the form [29]

$$
h(r)=c(r)+\rho \int \mathrm{d} \boldsymbol{r}^{\prime} c\left(\left|\boldsymbol{r}-\boldsymbol{r}^{\prime}\right|\right) h\left(r^{\prime}\right) .
$$

With $\tilde{h}(Q)$ denoting the Fourier transform of $h(r)$, the structure factor $S(Q)$ is defined as

$$
S(Q)=1+\rho \tilde{h}(Q) .
$$

The Ornstein-Zernike relation is exact. As it connects two unknown functions, one more relation or closure is needed in order to determine $g(r)$ and $c(r)$. Closures are approximate relations which arise from exact diagrammatic expansions of $g(r)$ in terms of $c(r)$ but with certain classes of diagrams ignored. The exact relation between $g(r)$ and $c(r)$ reads as [29]

$$
g(r)=\exp [-\beta v(r)+h(r)-c(r)+B(r)]
$$

where $v(r)$ is the pair potential and $B(r)$ is the so-called bridge function, consisting of the sum of all elementary diagrams that are not nodal. 
All known closures can be thought of as approximate relations for the form of $B(r)$. Common closures are the Percus-Yevick (PY) and hypernetted-chain (HNC) approximations [29]. In the PY closure, the approximation for $B(r)$ reads as

$$
B_{\mathrm{PY}}(r)=c(r)-h(r)+\ln [g(r)-c(r)]
$$

whereas in the HNC closure the approximation is made that the bridge function vanishes:

$$
B_{\mathrm{HNC}}(r)=0 \text {. }
$$

The PY closure is successful only for short-ranged, hard interactions and hence it will not be considered here. In addition to the HNC closure, we have considered two more closures of increasing degree of sophistication, the Rogers-Young (RY) closure [30] and the zeroseparation (ZSEP) closure [31-35]. The former (RY) closure reads as

$$
g(r)=\exp [-\beta v(r)]\left[1+\frac{\exp [\gamma(r) f(r)]-1}{f(r)}\right]
$$

where

$$
\gamma(r)=h(r)-c(r)
$$

and the 'mixing function' $f(r)$ is chosen to have the form

$$
f(r)=1-\exp (-\alpha r)
$$

The parameter $\alpha$ is determined such that thermodynamic consistency between the virial and compressibility pressures is achieved [29]. In the limit $\alpha=0$ one recovers the PY closure and in the opposite limit, $\alpha \rightarrow \infty$, the HNC closure.

The ZSEP closure includes three parameters and is a direct approximation for the bridge function having the form

$$
B_{\mathrm{ZSEP}}(r)=-\frac{\zeta \gamma^{2}(r)}{2}\left[1-\frac{\varphi \gamma(r)}{1+\alpha \gamma(r)}\right] .
$$

The ZSEP closure has been applied recently by Fernaud et al [9] to the PSM mentioned above, yielding excellent results for the correlation functions as compared to the simulation results. The three parameters $\zeta, \varphi$ and $\alpha$ are determined in such a way that virial-compressibility, Gibbs-Duhem and zero-separation consistencies are all fulfilled; for details on the above, we refer the reader to reference [9]. Evidently, for $\zeta=0$ the ZSEP closure reduces to the HNC closure.

In addition, in order to test the reliability of the various closures, we have performed standard NVT Monte Carlo (MC) simulations for different densities and temperatures. In what follows, we focus our attention on temperature $t=0.01$ on two grounds: on the one hand, according to the approximate phase diagram [16], at $t=0.01$ the system remains fluid at all densities and therefore we can study the development of the correlation functions with density for an unlimited range of the latter, without entering a region where the fluid is metastable. On the other hand, this temperature is low enough that significant structure is expected for the correlation functions of the fluid and hence the integral equation theories can be given a strong test. We present the results so obtained in figures 2, 3 and 4 and we discuss them below.

We begin with the RY closure. When the packing is not large enough, typically $\eta \lesssim 0.50$ at this temperature, the RY closure gives results which are in very good agreement with simulations, as can be seen in figures 2 and 3. However, above $\eta \approx 0.50$, the $g(r)$ from simulations starts penetrating towards $r=0$, which means physically that the probability of finding two particles 'sitting on top of each other' is finite and there is no 'correlation hole' 

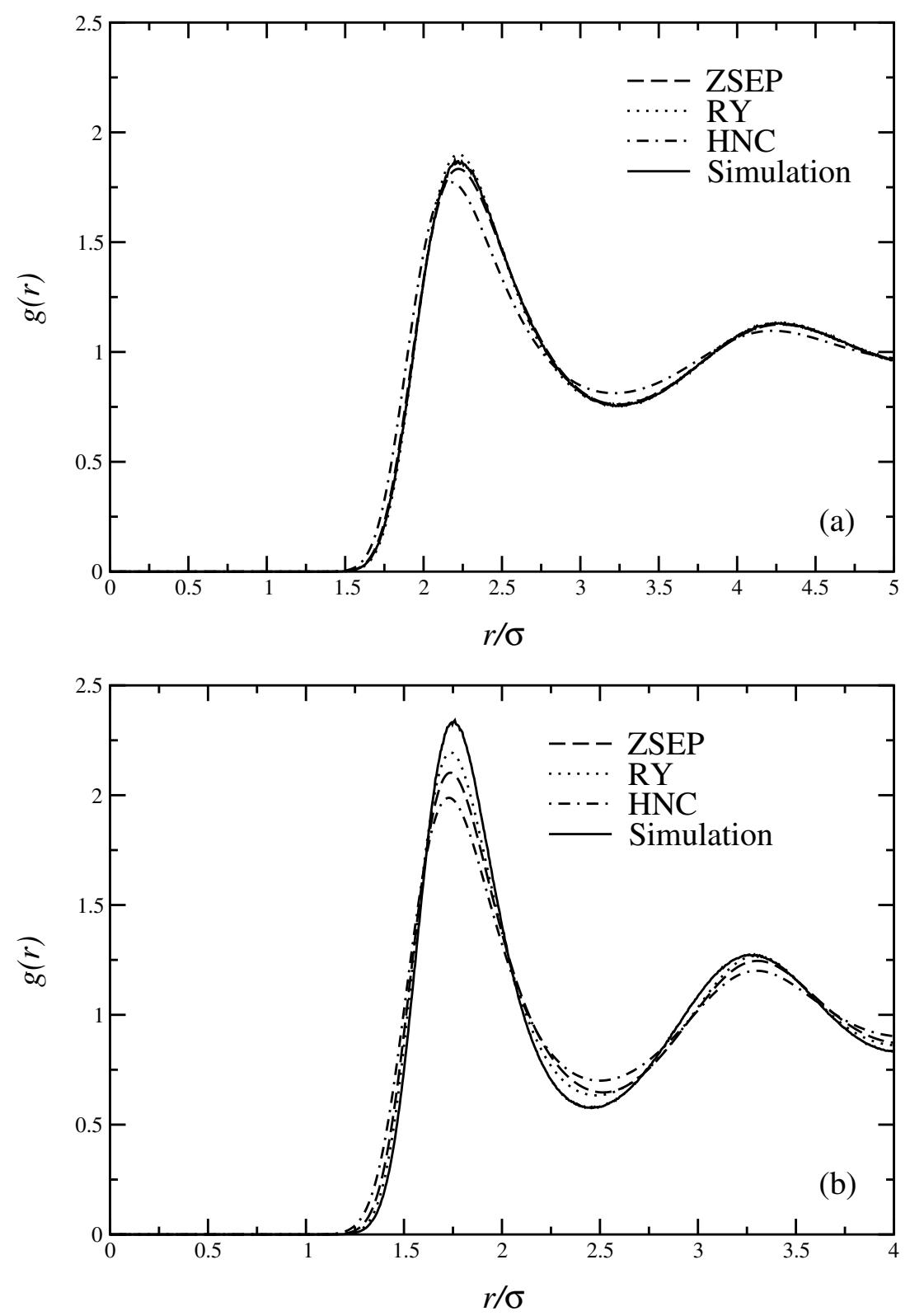

Figure 2. Comparison for the radial distribution function $g(r)$ between the simulation results and those obtained from the various closures, at $t=0.01$ and at small values of the packing fraction. (a) $\eta=0.05$; (b) $\eta=0.12$.

in $g(r)$. This is to be expected for a system with a bounded interaction. However, as can be seen from figure 4(a), the RY closure fails to capture precisely this penetration phenomenon, yielding $g(r)$ s that are too low at small separation and making the erroneous prediction that a correlation hole exists.

The reason for this behaviour can be traced back in the construction of the RY closure, equations (15) and (17), where it can be seen that the RY closure always looks like the PY 

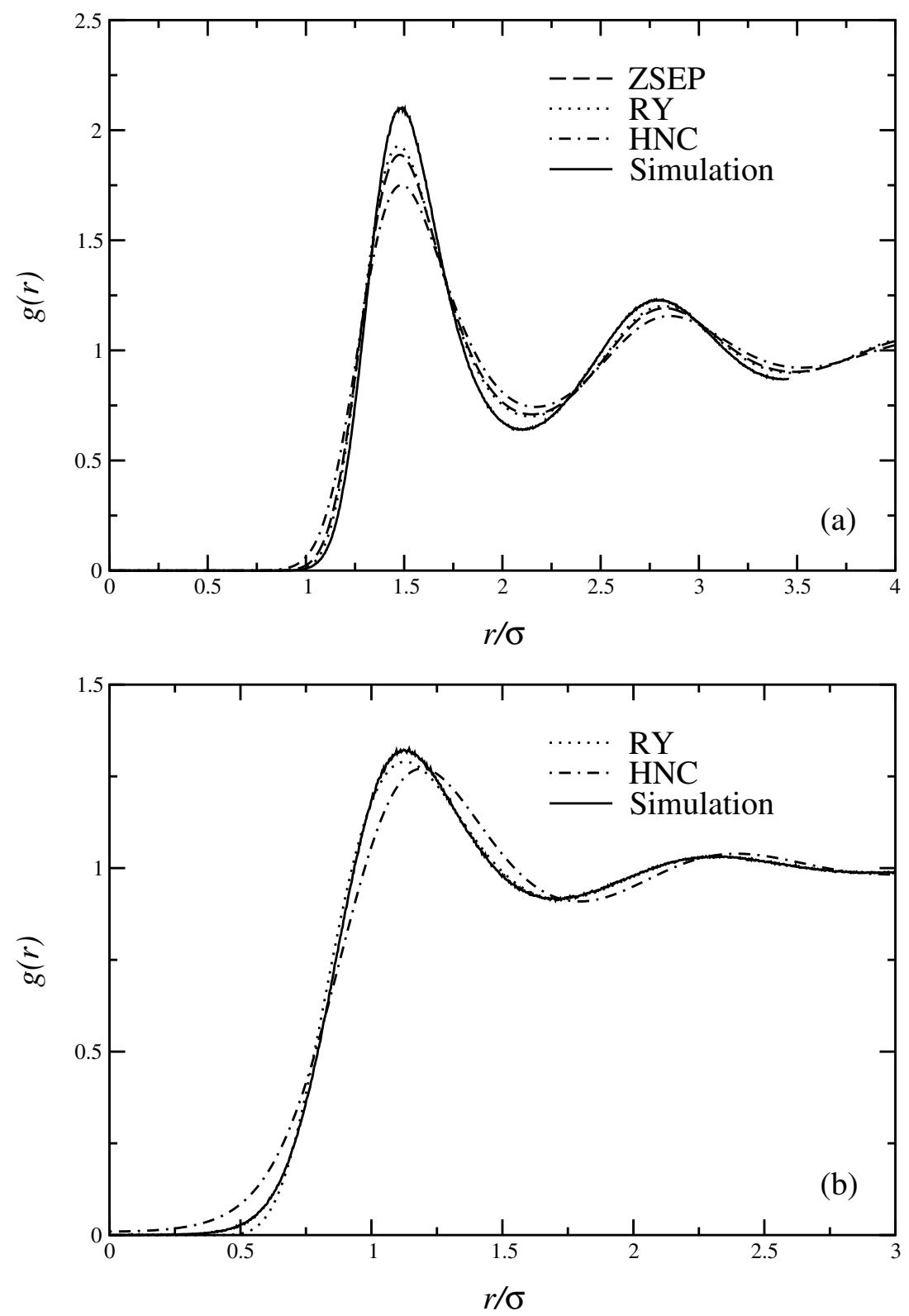

Figure 3. As figure 2, but for intermediate packings. (a) $\eta=0.20$; (b) $\eta=0.50$. Note the different scales of the vertical axes.

closure at small separations. The latter is however inaccurate for a long-range interaction lacking a hard core. We attempted to modify the RY closure by employing mixing functions yielding a HNC-like small- $r$ behaviour and a PY-like large- $r$ behaviour. However, this did not yield self-consistency parameters for the whole range of densities. Despite its inability to correctly describe the high-density penetration of $g(r)$, the standard RY closure yields nevertheless a self-consistency parameter $\alpha$ for all densities considered here. In addition, this parameter grows with density, thus pointing to a tendency of the RY closure to reduce to the 

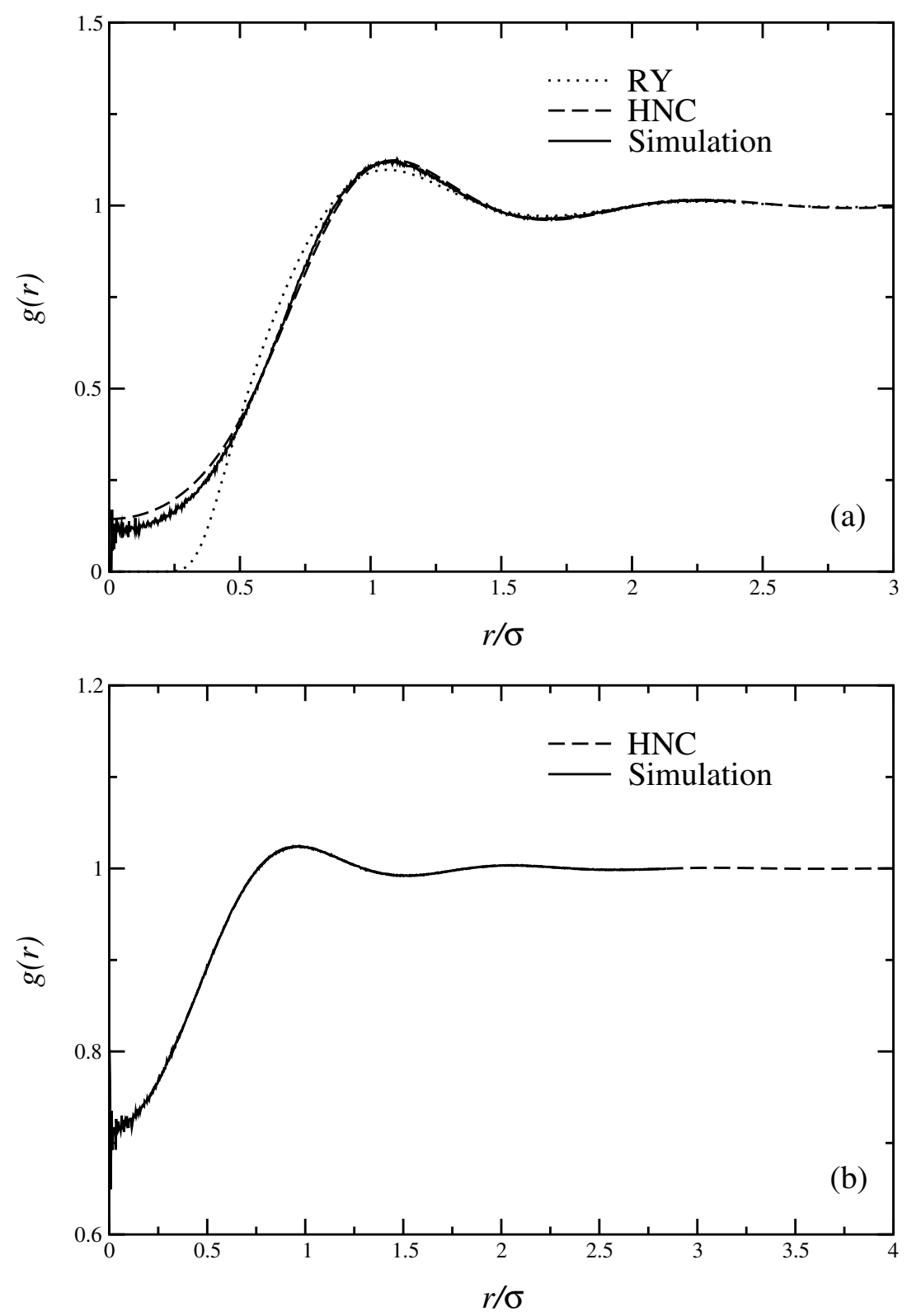

Figure 4. As figure 2, but for high packings. (a) $\eta=1.00$; (b) $\eta=6.00$, where the simulation result is indistinguishable from the $\mathrm{HNC}$ result.

HNC closure at high packings.

In view of the failure of the RY closure, we are led to consider the ZSEP closure which has precisely the property that in its implementation the value of $g(r)$ at zero separation plays an important role and is determined self-consistently. In fact, the resounding success of the ZSEP closure in describing the $g(r)$ of the PSM (also a bounded interaction) has been mainly attributed to this property [9]. As can be seen from figures 2 and 3(a), the ZSEP closure performs only slightly less well than the RY closure up to a packing fraction $\eta \approx 0.25$. The 
self-consistency parameters $\zeta, \varphi$ and $\alpha$ of the ZSEP closure are displayed in figure 5. The parameter $\zeta$ which appears as an overall factor in the parametrization of the bridge function (see equation (18)) decreases with density and close to $\eta=0.25$ it is small enough and the ZSEP closure practically reduces to the HNC closure.

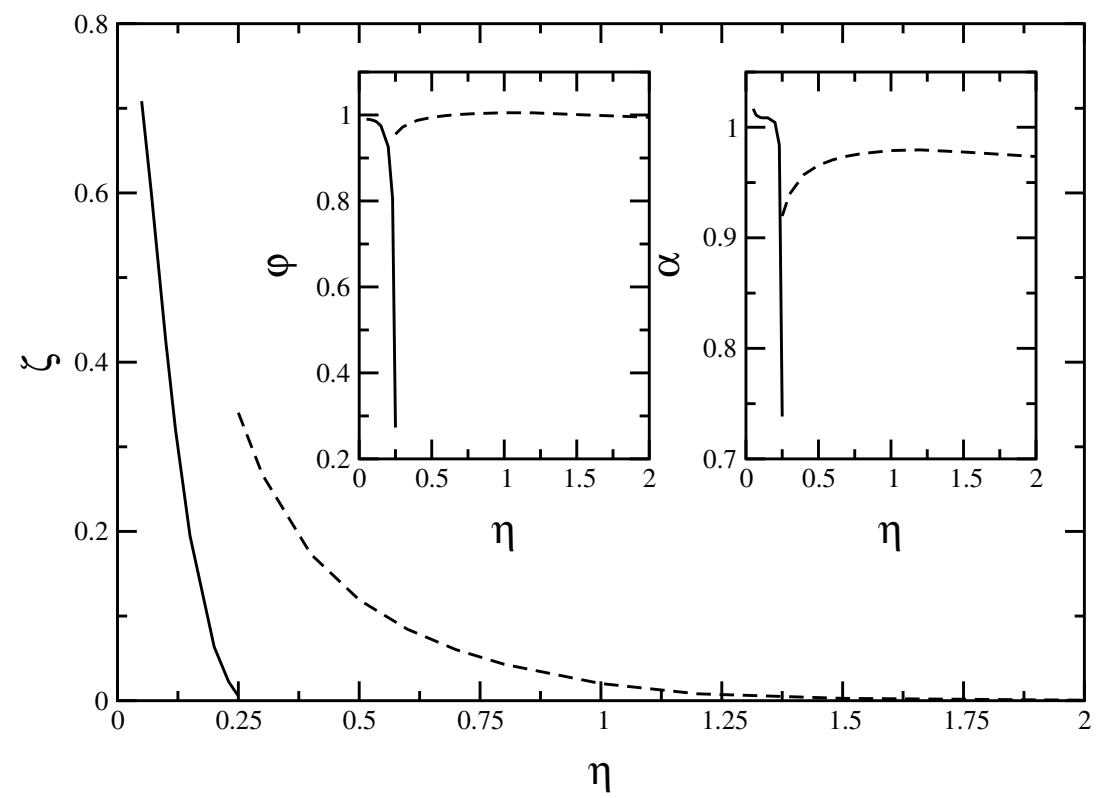

Figure 5. The self-consistency parameters of the ZSEP closure applied to the Gaussian core model at reduced temperature $t=0.01$ as functions of the packing fraction $\eta$. The solid lines denote the physical branch and the dashed lines the unphysical branch (see the text).

However, at packing fractions $\eta>0.25$, a second branch of solutions appears, which is denoted by the dashed lines in figure 5. This branch is disconnected from the first and hence it causes the $g(r)$ to behave discontinuously at this packing fraction, a result which is clearly unphysical. This second branch produces $g(r)$ s that show too much penetration and too little structure, when compared with the simulation results. The reason for this unphysical behaviour can be traced to the fact that this second branch corresponds to bridge functions which are positive at small separations $r$. Indeed, from equation (18), and taking into account that $\gamma(r) \gg 1$ at small separations, we can see that $B_{\mathrm{ZSEP}}(r)$ has the sign of the product $\zeta(\varphi-\alpha) / \alpha$. For the first, physical branch (solid lines in figure 5) this combination is negative because $\varphi<\alpha$. For the second, unphysical branch, this combination is positive because $\varphi>\alpha$ there. A positive bridge function acts then as an additional 'attractive potential' in equation (12) and causes the overpenetration in $g(r)$ mentioned above.

The appearance of a branch of solutions of the ZSEP closure for which the bridge function is positive is a sign of internal inconsistency of the closure and in this sense the ZSEP closure signals its own limits of applicability. Indeed, the bridge function of any system has been shown to be a quasi-universal function, which can always be mapped onto the bridge function of a suitably defined hard-sphere system having a hard-sphere diameter that depends on the characteristics of the interaction and the thermodynamic point under consideration [36]. The bridge function of the HS system is, however, practically exactly known and it is essentially a negative function for all $r$. Hence, a positive bridge function is physically unacceptable and the second branch of solutions of the ZSEP closure has to be discarded. The results from 
this second branch come again into very good agreement with simulation at packing fractions above $\eta \approx 1.00$ because, as seen in figure 5, the parameter $\zeta$ is already very small there and the bridge function has a negligible contribution to $g(r)$; even the unphysical branch reduces to the HNC one at high densities. However, there is no way to bridge the physical solutions at packings $\eta \leqslant 0.25$ with the HNC-like solutions within the ZSEP closure, that is without having to compare with independently produced simulation results.

We comment next on the quality of the HNC closure. As can be seen from figures 2-4, the HNC closure underestimates the structure at small-to-intermediate packings but yields otherwise reasonable results. It does not suffer from any of the problems of the more sophisticated closures and, in fact, it seems to be the best at high densities. In order to further explore this property (which is supported by the fact that the other two closures tend to the HNC one at this limit), we also solved this closure at extremely high packing fractions. Here, the highest packing at which we simulated was $\eta=6.00$, due to time constraints. With increasing $\eta$, a very large number of particles would be required in the simulation box in order to obtain reliable results. In view of the fact that the HNC closure gives results which coincide with those from Monte Carlo simulation at $\eta=6.00$ (see figure 4(b)), we gain confidence at this closure and apply it for arbitrarily high densities, in order to obtain information on the structure of the very dense fluid. In figure 6(a), we show results for $g(r)$, where it can be seen that at very high packings $g(r)$ tends to unity and hence $h(r)=g(r)-1$ tends to zero. However, this does not mean that the structure factor $S(Q)$ tends to unity as well, as a naive guess based on the definition $S(Q)=1+\rho \tilde{h}(Q)$ would imply. The quantity $\tilde{h}(Q)$ tends to zero, but at the same time the prefactor $\rho$ diverges, thus giving rise to a non-trivial $S(Q)$.

Results for the corresponding structure factor $S(Q)$ are shown In figure 6(b). It can be seen that, for high densities, the peak of $S(Q)$ disappears and the latter looks like a 'smoothed step function' with values ranging from zero to one. The value of $Q$ at which the crossover occurs does not scale as a power law of the density but rather shifts to the right by almost a constant every time the packing fraction is increased by an order of magnitude. This hints at a very weak dependence of this crossover value on the density; we return to this point in section 4.

The liquid-state correlation functions of the GCM display an anomalous behaviour in comparison with that of 'normal' liquids, interacting by means of hard, unbounded interactions. For the latter, the structure grows monotonically with increasing density and eventually the systems freeze. Here, the structure grows up to a packing fraction $\eta \cong 0.12$ at $t=0.01$ and for higher densities it becomes weaker again. To amply demonstrate this phenomenon, we show in figure 7 the structure factors obtained from the MC simulations for a wide range of densities, where it can be seen that the height of the peak of $S(Q)$ attains its maximum value at $\eta \cong 0.12$. The same phenomenon has been observed in the liquid structure of star polymer solutions employing the interaction potential given by equation (1) [4,5,37] and, in fact, for star polymer solutions the effect has also been observed experimentally [38].

This behaviour of $S(Q)$ is closely related to re-entrant melting [3]. Indeed, the height of the maximum of $S(Q)$ is a diagnostic tool for the freezing transition. According to the Hansen-Verlet criterion [39,40], a liquid crystallizes when $S(Q)$ at its highest peak has the quasi-universal value 2.85. At higher values of the peak the system is solid and at lower values it is fluid. The evolution of $S(Q)$ with density shown in figure 7 in conjunction with the HansenVerlet criterion implies that the system approaches crystallization at about $\eta=0.12$ and then remelts. The height of the peak at $\eta=0.12$, which is indeed slightly above the Hansen-Verlet value, implies that at this temperature the system barely freezes and that $t=0.01$ is very close to the upper freezing temperature. We will confirm this prediction in section 6 , where we will also use the structural results obtained here in order to calculate the free energy of the fluid. 

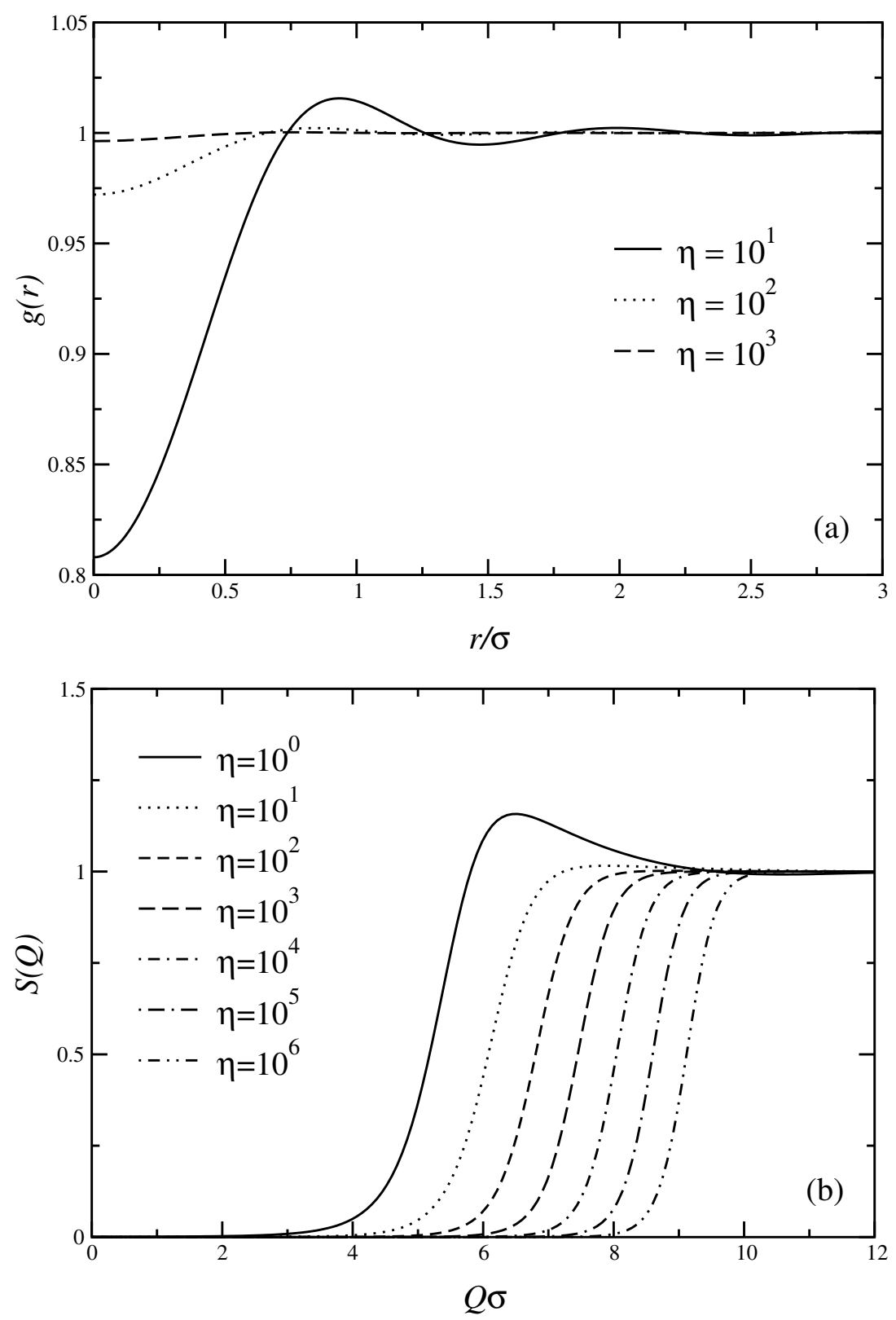

Figure 6. (a) The radial distribution function $g(r)$ and (b) the structure factor $S(Q)$ as obtained by the HNC closure for extremely high values of the packing fraction.

Finally, we have performed MC simulations and solved the HNC closure also at much higher temperatures, $t=1.00$, corresponding to the physical domain for the effective interactions between polymer chains. There, we found that the HNC closure accurately reproduces the simulation results at all densities and that the liquid has very little structure, a result which can be easily understood in view of the fact that the thermal energy, which is equal to the interaction strength there, washes out the correlation effects caused by the interactions. 


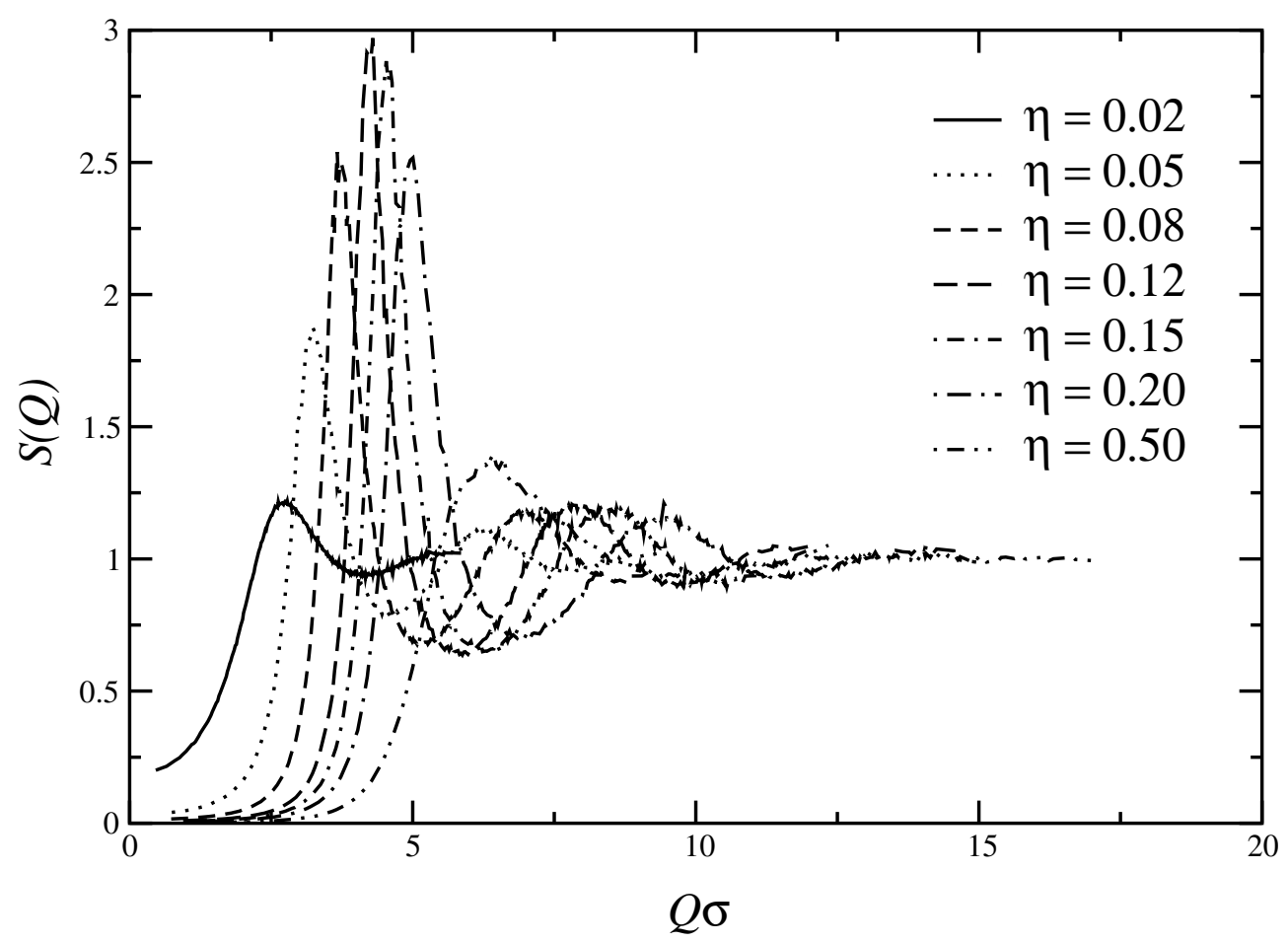

Figure 7. The structure factor $S(Q)$ of the GCM at $t=0.01$ for different packing fractions, as obtained by Monte Carlo simulation.

\section{The high-density limit in the fluid state}

The results of the preceding section on the structure of the fluid at high densities indicate that the correlations are becoming weaker as density grows and that the system approaches some kind of 'infinite-density ideal-gas' limit, where $g(r)=1$. This limit was assumed already by Stillinger [11]. There, the internal energy of a high-density fluid was approximated by that of a random distribution of points interacting by means of the Gaussian potential, i.e., the positions of the points were assumed to be uncorrelated. The relation $g(r)=1$ was used explicitly there in deriving an estimate for the internal energy of the high-density fluid and it was shown that in fact the complete absence of a correlation hole in the fluid raises its internal energy with respect to that of a solid (where a correlation hole is necessarily present) by exactly $\varepsilon / 2$ per particle. Yet, the existence of this ideal-gas limit was not proven. Here, we are going to present a density functional mean-field theory which establishes this limit and provides analytic expressions for the correlation functions of the GCM fluid at high densities.

In the framework of density functional theory (DFT), one considers in general a system with a spatially modulated one-particle density $\rho(\boldsymbol{r})$. The Helmholtz free energy of the system is then a unique functional of the one-particle density [41], $F=F[\rho(\boldsymbol{r})]$, and can be written as a sum of an entropic, ideal part $F_{\text {id }}[\rho(\boldsymbol{r})]$ which is exactly known and an excess part $F_{\text {ex }}[\rho(\boldsymbol{r})]$ which is in general unknown:

$F[\rho(\boldsymbol{r})]=F_{\mathrm{id}}[\rho(\boldsymbol{r})]+F_{\mathrm{ex}}[\rho(\boldsymbol{r})]=k_{B} T \int \mathrm{d} \boldsymbol{r} \rho(\boldsymbol{r})\left[\ln \left[\rho(\boldsymbol{r}) \Lambda^{3}\right]-1\right]+F_{\mathrm{ex}}[\rho(\boldsymbol{r})]$.

In equation (19) above, $\Lambda$ denotes the thermal de Broglie wavelength of the particles. 
We consider the limit $\rho \sigma^{3} \gg 1$. The average interparticle distance $a \equiv \rho^{-1 / 3}$ becomes vanishingly small in this limit and $a \ll \sigma$ holds, i.e., the potential is extremely long range. Every particle is simultaneously interacting with an enormous number of neighbouring molecules and in the absence of short-range excluded-volume interactions the excess free energy of the system can be approximated by a simple mean-field term, equal to the internal energy of the system:

$$
F_{\mathrm{ex}}[\rho(\boldsymbol{r})] \cong \frac{1}{2} \iint \mathrm{d} \boldsymbol{r} \mathrm{d} \boldsymbol{r}^{\prime} v\left(\left|\boldsymbol{r}-\boldsymbol{r}^{\prime}\right|\right) \rho(\boldsymbol{r}) \rho\left(\boldsymbol{r}^{\prime}\right)
$$

with the approximation becoming more accurate with increasing density and eventually exact at $\bar{\rho} \rightarrow \infty$. The direct correlation function $c\left(\left|\boldsymbol{r}-\boldsymbol{r}^{\prime}\right| ; \rho\right)$ of a fluid of density $\rho$ is given by the second functional derivative of $F_{\mathrm{ex}}[\rho(\boldsymbol{r})]$ with respect to the density [41], namely

$$
c\left(\left|\boldsymbol{r}-\boldsymbol{r}^{\prime}\right| ; \rho\right)=-\lim _{\rho(\boldsymbol{r}) \rightarrow \rho} \frac{\delta^{2} \beta F_{\mathrm{ex}}[\rho(\boldsymbol{r})]}{\delta \rho(\boldsymbol{r}) \delta \rho\left(\boldsymbol{r}^{\prime}\right)} .
$$

Combining equations (20) and (21), we find that the dcf of the system at high densities becomes independent of $\rho$ and is simply proportional to the interaction:

$$
c(r)=-\beta v(r) \text {. }
$$

Equation (22) above has a strong similarity with the mean-spherical approximation (MSA) $[29,42]$, introduced as a perturbation theory to study systems interacting by potentials that can be separated into a hard-sphere interaction (diameter $\left.\sigma_{\mathrm{HS}}\right) v_{0}(r)$ and a 'soft tail' $\phi(r)$. In the MSA, the radial distribution function of the system $g(r)$ vanishes for $r<\sigma_{\mathrm{HS}}$ and the direct correlation function $c(r)$ at $r>\sigma_{\mathrm{HS}}$ is given by $c(r)=-\beta \phi(r)$. The main difference from the MSA here is that there is no reference potential $v_{0}(r)$ because there are no hard cores in the system. Hence, equation (22) holds with the total interaction on the right-hand side and for the whole range of separations $r$. Moreover, unlike the MSA which is essentially a hightemperature approximation, equation (22) holds for the whole temperature range, provided that the density is high enough $\left(\rho \sigma^{3} \gg 1\right)$. Of course, the mean-field approximation becomes also valid at high temperatures $(t \gg 1)$ irrespective of the density, because there the thermal energy completely dominates over the bounded interaction. This happens in contradistinction with diverging interactions, where short-range correlation effects always survive, at all temperatures.

The Fourier transform $\tilde{c}(Q)$ of the dcf is obtained easily from equations (22) and (2), and for the GCM it has the form

$$
\tilde{c}(Q)=-\pi^{3 / 2} \beta \varepsilon \sigma^{3} \exp \left[-(Q \sigma / 2)^{2}\right] .
$$

Using the Ornstein-Zernike equation and the ensuing relation $S(Q)=[1-\rho \tilde{c}(Q)]^{-1}$ we obtain for the structure factor of the GCM the expression

$$
S(Q)=\frac{1}{1+\pi^{3 / 2} \beta \varepsilon \rho \sigma^{3} \exp \left[-(Q \sigma / 2)^{2}\right]} .
$$

This analytic expression is compared with the MC result at $\beta \varepsilon=100(t=0.01)$ and $\eta=6.00$ in figure 8 . The excellent agreement between the two demonstrates the validity of the simple mean-field theory at high densities.

Equation (24) shows that at high densities $S(Q)$ is a monotonic function of $Q$ and has the shape of a 'smoothed step function' with values ranging from $\left(\pi^{3 / 2} \beta \varepsilon \rho \sigma^{3}\right)^{-1} \cong 0$ at low $Q$ to unity at high $Q$. The crossover between the two regimes occurs at a characteristic wavenumber $Q_{*}$ at which $S(Q)=1 / 2$ and which, according to equation (24), is given by

$$
Q_{*} \sigma=2 \sqrt{\ln \left(\pi^{3 / 2} \beta \varepsilon \rho \sigma^{3}\right)} .
$$




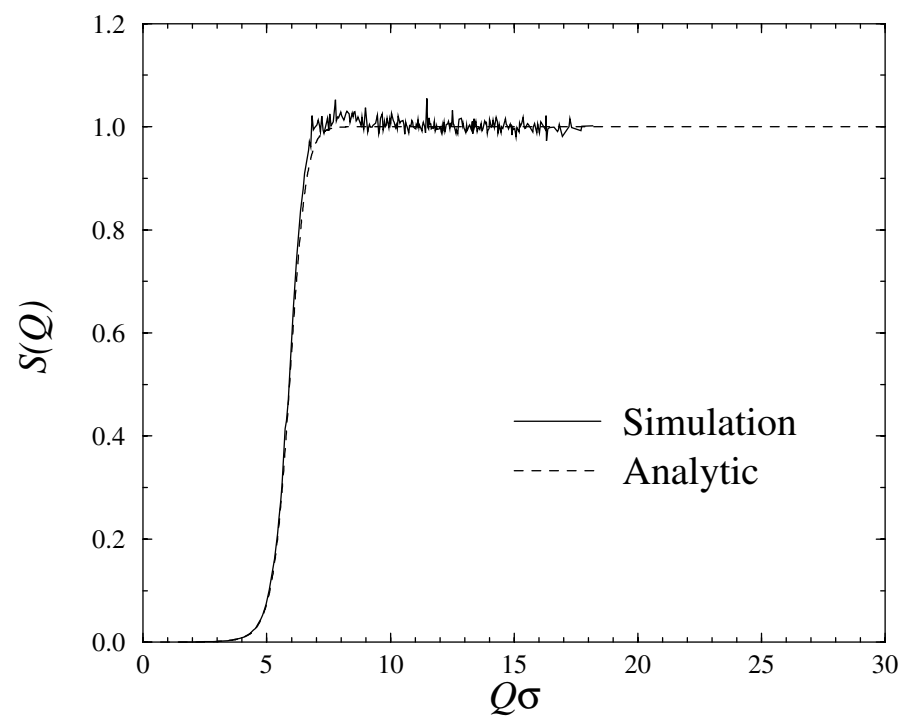

Figure 8. The structure factor $S(Q)$ of the GCM at $t=0.01$ and $\eta=6.00$ as obtained from simulation (solid line) and as given by the analytical expression, equation (24) (dashed line).

Note the very weak, square-root-logarithmic dependence of $Q_{*}$ on density and the inverse temperature.

Another quantity of interest is the isothermal compressibility $\chi_{T}$ of the system, defined as

$$
\chi_{T}=\left(V \frac{\partial^{2} F}{\partial V^{2}}\right)^{-1}
$$

where $F$ is the Helmholtz free energy, and also related to the $Q \rightarrow 0$ limit of $S(Q)$ through [29]

$$
\rho k_{B} T \chi_{T}=S(0) \text {. }
$$

The expressions (19) and (20) yield the Helmholtz free energy in the high-density limit as

$$
F=k_{B} T N\left[\ln \left(\rho \Lambda^{3}\right)-1\right]+\frac{1}{2} N \pi^{3 / 2} \varepsilon \rho \sigma^{3}
$$

and from equations (26) and (28) we obtain

$$
\chi_{T}=\frac{1}{k_{B} T \rho+\pi^{3 / 2} \varepsilon \rho^{2} \sigma^{3}}
$$

which evidently satisfies the compressibility sum rule (27) with $S(Q)$ given by equation (24). We note that at high densities $\chi_{T}$ obeys the scaling

$$
k_{B} T \chi_{T} \sim t \rho^{-2} .
$$

Moreover, from equation (28) the pressure $P=-(\partial F / \partial V)$ is obtained as

$$
P=k_{B} T \rho+\frac{1}{2} \pi^{3 / 2} \varepsilon \rho^{2} \sigma^{3} .
$$

Since $S(Q)=1+\rho \tilde{h}(Q)$, equation (24) immediately yields an analytic expression for the Fourier transform $\tilde{h}(Q)$ of the correlation function $h(r)$, namely

$$
\tilde{h}(Q)=-\frac{\pi^{3 / 2} \beta \varepsilon \sigma^{3} \exp \left[-(Q \sigma / 2)^{2}\right]}{1+\pi^{3 / 2} \beta \varepsilon \rho \sigma^{3} \exp \left[-(Q \sigma / 2)^{2}\right]} .
$$


At low $Q$, where the exponential is of order unity, the term proportional to the density in the denominator dominates and $\tilde{h}(Q)$ behaves as $-\rho^{-1} \rightarrow 0$ as $\rho \rightarrow \infty$. At high $Q$, the exponential itself tends to zero. Hence, the function $\tilde{h}(Q)$ vanishes as $\rho \rightarrow \infty$ with the leading term being proportional to $\rho^{-1}$. Consequently, the correlation function $h(r)$ vanishes as well and $g(r) \rightarrow 1$ as $\rho \rightarrow \infty$. This is the particular 'high-density ideal-gas' limit of the model. However, a clear distinction must be drawn between this 'interacting ideal gas' and the usual ideal gas, in which either the system is non-interacting or an interacting system is considered at the opposite limit, $\rho \rightarrow 0$. In the usual ideal-gas limit, we have $c(r)=\exp [-\beta v(r)]-1$, $S(Q)=1$ and $g(r)=\exp [-\beta v(r)]$. The ideal-gas pressure $P$ scales linearly with the density and the ideal compressibility $\chi_{T}$ scales with $\rho^{-1}$. Here, $c(r)=-\beta v(r), S(Q)$ is not unity in the whole $Q$-range, the pressure scales as $\rho^{2}$ (see equation (31)) and the isothermal compressibility as $\rho^{-2}$ (see equation (30)). Nevertheless, the above considerations point to a kind of interesting duality of the GCM in the liquid phase, in which the system is trivially ideal at low densities and becomes ideal again (vanishing correlations) at high densities. This can be thought of as the counterpart for the fluid state of the duality discovered by Stillinger in the crystalline state [15].

The mean-field approximation put forward in this section is not limited to the Gaussian potential. It should be valid for all interactions $v(r)$ which are finite, analytic functions and which tend to zero fast enough at infinite separations that the thermodynamic functions (e.g., the internal energy) are extensive and the Fourier transform of $v(r)$ exists. Hence, we make the theoretical prediction that this peculiar high-density ideal-gas limit should exist for all potentials satisfying the requirements put forward above. In addition, it can now be understood why the HNC closure showed the best agreement with simulation results among all closures at high densities. As $g(r) \rightarrow 1, h(r) \rightarrow 0$ and $c(r) \rightarrow-\beta v(r)$ in this limit, the exact relation (12) forces the bridge function $B(r)$ to vanish. For bounded, analytic potentials decaying fast enough to zero, the HNC closure becomes exact at high densities.

\section{The solid: Einstein model}

In this section we present the approach employed for the calculation of the free energies of candidate crystalline states of the model, necessary for determining the phase diagram of the GCM. As we are dealing with a soft interaction, a harmonic approximation in the solid is justified and we adopt the simple Einstein model $[43,44]$ as a means to estimate the free energy of the latter. The approach is based on the Gibbs-Bogoliubov inequality [29]. The latter states that the Helmholtz free energy $F$ of a system having Hamiltonian $\mathcal{H}$ is related to the Helmholtz free energy $F_{0}$ of a reference system having Hamiltonian $\mathcal{H}_{0}$ by

$$
F \leqslant F_{0}+\left\langle\mathcal{H}-\mathcal{H}_{0}\right\rangle_{0}
$$

where the canonical average on the right-hand side is taken in the reference system. The procedure is useful if

(i) a simple enough reference Hamiltonian can be chosen which physically corresponds to a situation close enough to the real one and in which $F_{0}$ and the average $\left\langle\mathcal{H}-\mathcal{H}_{0}\right\rangle_{0}$ can be calculated in a straightforward way and

(ii) this Hamiltonian contains at least one variational parameter which can be chosen so as to minimize the right-hand side, obtaining in this way a lower upper bound for the true free energy of the system. 
In a harmonic solid, a reasonable non-interacting reference system is the Einstein solid, characterized by the Hamiltonian

$$
\mathcal{H}_{0}=\sum_{i=1}^{N}\left[\frac{\boldsymbol{p}_{i}^{2}}{2 m}+\frac{k}{2}\left(\boldsymbol{r}_{i}-\boldsymbol{R}_{i}\right)^{2}\right]
$$

where $\boldsymbol{p}_{i}$ is the canonical momentum of a particle of mass $m, k$ is the 'spring constant' which plays the role of a variational parameter and the set $\left\{\boldsymbol{R}_{i}\right\}$ forms a prescribed Bravais lattice. For the GCM, the real Hamiltonian reads as

$$
\mathcal{H}=\sum_{i=1}^{N} \frac{\boldsymbol{p}_{i}^{2}}{2 m}+\sum_{i=1}^{N} \sum_{j=i+1}^{N} \varepsilon \exp \left(-\frac{\left|\boldsymbol{r}_{i}-\boldsymbol{r}_{j}\right|^{2}}{\sigma^{2}}\right) .
$$

The calculation of the Helmholtz free energy of the Einstein solid is a trivial exercise yielding

$$
\frac{F_{0}}{N}=\frac{3}{2} k_{B} T \ln \left(\frac{\tilde{\alpha}}{\pi}\right)+3 k_{B} T \ln \left(\frac{\Lambda}{\sigma}\right)
$$

where

$$
\tilde{\alpha}=\frac{\beta k \sigma^{2}}{2} .
$$

The one- and two-particle densities in the reference system are given by a sum of Gaussians and a double sum of products of Gaussians, respectively, where the latter are centred on lattice sites and the sums are carried upon all these sites. As the interaction has itself a Gaussian form, the calculation of the quantity $\left\langle\mathcal{H}-\mathcal{H}_{0}\right\rangle_{0}$ reduces to integrals that can be solved analytically. The final result reads as

$$
\frac{\left\langle\mathcal{H}-\mathcal{H}_{0}\right\rangle_{0}}{N}=\frac{1}{2} \sum_{j \neq 0} n_{j}\left(\frac{\tilde{\alpha}}{\tilde{\alpha}+2}\right)^{3 / 2} \varepsilon \exp \left(-\frac{\tilde{\alpha} X_{j}^{2}}{\tilde{\alpha}+2}\right)-\frac{3 k_{B} T}{2} .
$$

The sum on the rhs is carried over all shells of lattice vectors, i.e., sets of lattice vectors of equal length, with the shell $j=0$ (self-interaction) excluded. The quantity $n_{j}$ is the number of lattice vectors belonging to a shell and $X_{j}=\left|\boldsymbol{R}_{j}\right| / \sigma$. It can be easily seen that at $T=0$, where $\tilde{\alpha} \rightarrow \infty$, the first term on the rhs of equation (38) reduces to the internal energy per particle of the crystalline arrangement considered, the lattice sum. At zero temperature there is no variational parameter and the Einstein model becomes exact; the winning phase is the one with the lowest lattice energy. At finite temperatures, the sum of the terms on the rhs of equations (36) and (38) has to be minimized with respect to $\tilde{\alpha}$ for any given lattice structure. The minimum comes about through the competition of the entropic, logarithmic term on the rhs of equation (36), which favours delocalization at $\tilde{\alpha}=0$, and the internal energy term on the rhs of equation (38), which favours localization at $\tilde{\alpha} \rightarrow \infty$. The value obtained is then the estimate for the Helmholtz free energy of the given lattice and the procedure can be repeated for every candidate lattice. We note that the term $3 k_{B} T \ln (\Lambda / \sigma)$ on the rhs of equation (36) can be dropped because it occurs for all possible phases of the system, fluid and solid, and does not affect the free-energy comparisons between them.

We have performed the minimization for various candidate lattice structures: fcc, bcc, simple cubic, diamond and body-centred orthogonal, in which the ratios between the lattice constants of the conventional unit cells were used as additional variational parameters $[3,4]$. We always assumed a lattice with a single occupancy per site, in view of the result of Stillinger stating that solids with multiple occupancies are indeed unstable [11]. For the whole range of temperatures $0 \leqslant t \leqslant 0.015$ considered, we always found the fcc and bcc to be the only stable crystals, with the former winning at low densities and the latter at high densities. The quantitative results are given in section 6 . 


\section{The quantitative phase diagram}

In this section we switch from the variable $\eta$ to the variable $\bar{\rho} \equiv \rho \sigma^{3}$ as a measure of the density, in order to allow for a direct comparison with the approximate results of Stillinger and Stillinger [16]. With the free energy of the crystals obtained by the procedure outlined in section 5, the phase diagram can be drawn if the corresponding free energy of the fluid is also known. The latter can be obtained from the results of the integral equation theories outlined in section 3 .

The Helmholtz free energy of the liquid is the sum of the ideal and excess terms (see section 4), namely

$$
F(\rho, T)=N k_{B} T\left[\ln \left(\rho \sigma^{3}\right)-1\right]+3 N k_{B} T \ln (\Lambda / \sigma)+F_{\text {ex }}(\rho, T) .
$$

If for a fixed temperature the radial distribution function $g(r)$ is known for a region of densities, then one possibility for calculating the free energy is through the so-called virial route. Here, one calculates for every density the excess pressure $P_{\mathrm{ex}}$ of the system via

$$
P_{\mathrm{ex}}=-\frac{2 \pi \rho^{2}}{3} \int_{0}^{\infty} r^{3} v^{\prime}(r) g(r) \mathrm{d} r
$$

where $v^{\prime}(r)=\mathrm{d} v(r) / \mathrm{d} r$. Then, the excess free energy can be calculated by integrating the thermodynamic relation $P_{\mathrm{ex}}=-\partial F_{\mathrm{ex}} / \partial V$ from $\rho=0$ up to the given density, under the initial condition $F_{\mathrm{ex}}(\rho=0, T)=0$. Alternatively, one can use the results for the structure factor $S(Q)$ and the definition of the isothermal compressibility $\chi_{T}$ (equations (26) and (27)) to calculate the liquid free energy, following in this way the compressibility route.

If thermodynamic consistency between the two routes is not explicitly enforced in an otherwise approximate closure, then the results from two routes are different, a problem known as thermodynamic inconsistency of the closure [29]. The RY and ZSEP closures are thermodynamically consistent but the HNC closure is not. However, as explained in section 3, neither the RY nor the ZSEP closure yield reliable results for the whole density range. If we were dealing with a usual system, without re-entrant melting, then we could have used the RY or the ZSEP results at low or intermediate densities. However, we are interested also in the high-density-fluid free energies, where in fact the $\mathrm{HNC}$ closure becomes exact. A combination of low-density results from one closure and high-density results from another is not of much use either, because it would produce unphysical discontinuities in the free energy or its derivatives at the point of switching between the two. We are thus led to employ the HNC closure in the whole density domain in order to perform the thermodynamic integration and obtain the fluid free energy.

The HNC compressibility route yields at low densities fluid free energies that are too low, leading to the erroneous result that the HS-like freezing of the Gaussian fluid into a fcc lattice does not take place. There are two factors playing an important role here: on the one hand, the predicted isothermal compressibilities are too high, causing a fluid free energy which is too low, and on the other hand, the solid free energy, being a product of the variational procedure outlined in section 5, is unavoidably higher than the true one; see equation (33). It is therefore pertinent to follow the $\mathrm{HNC}$ virial route in calculating the fluid free energy. The latter leads in fact to an overestimation of the fluid free energy, but this compensates for the overestimation of the solid free energy and leads to the physically correct picture of freezing into an fcc solid at the low-density part of the phase diagram. We have thus calculated the fluid free energies through the HNC virial route for a range of temperatures $10^{-5} \leqslant t \leqslant 0.015$ for a range of densities $0 \leqslant \rho \sigma^{3} \leqslant 1.00$ and performed the common-tangent construction on the resulting $F_{\text {fluid }}(\rho) / V$ and $F_{\text {solid }}(\rho) / V$ curves to obtain the phase boundaries. The resulting phase diagram is shown in figure 9. 


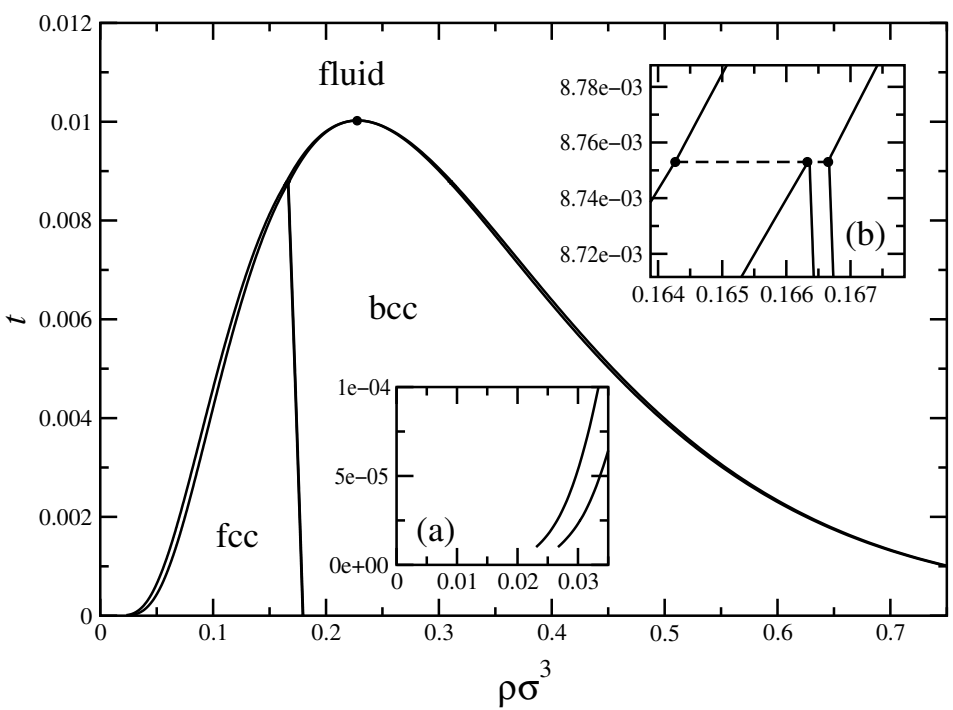

Figure 9. The phase diagram of the GCM obtained by the approach described in the text. The fcc-bcc coexistence lines are also double lines but they cannot be resolved on the scale of the figure because the fcc-bcc density gap is too small. The full dot marks the point at which the fluid-bcc coexistence curves turn around. The two insets show details of the phase diagram. (a) In the neighbourhood of zero densities and temperatures. (b) In the neighbourhood of the fluid-fcc-bcc triple temperature, with the dashed line denoting the triple line between these coexisting phases.

The phase diagram obtained is very similar to the approximate one drawn by Stillinger and Stillinger [16]. It shows the sequence of freezing, structural ( $\mathrm{fcc} \rightarrow \mathrm{bcc}$ ) and remelting transitions as well as the upper freezing temperature $t_{\mathrm{u}}$ associated with the corresponding density $\bar{\rho}_{\mathrm{u}}$. The coordinates of this point, where the fluid-bcc coexistence lines turn around, are $\left(t_{\mathrm{u}}, \bar{\rho}_{\mathrm{u}}\right)=(0.0102,0.2292)$. This is in perfect agreement with the preliminary results from section 3 , where at $t=0.01$ the structure factor at $\eta=0.12$, corresponding to $\bar{\rho}=0.2292$, was found to slightly exceed the Hansen-Verlet value. The fcc-bcc coexistence lines run linearly from the points $\bar{\rho}_{\mathrm{fcc}}=0.17941$ and $\bar{\rho}_{\mathrm{bcc}}=0.17977$ at $t=0$ to the points $\bar{\rho}_{\mathrm{fcc}}=0.16631$ and $\bar{\rho}_{\text {bcc }}=0.16667$ at the triple temperature $t_{\mathrm{t}}=8.753 \times 10^{-3}$. The density gap between the fcc and bcc coexisting densities remains constant and equal to $\Delta \bar{\rho}=3.6 \times 10^{-4}$. The density of the coexisting fluid at the triple temperature is $\bar{\rho}_{\text {fluid }}=0.16431$.

It should be emphasized that, notwithstanding its deceptive appearance in figure 9 , the point $\left(t_{\mathrm{u}}, \bar{\rho}_{\mathrm{u}}\right)$ is not a critical point [11]. At $\left(t_{\mathrm{u}}, \bar{\rho}_{\mathrm{u}}\right)$, two common tangents between the fluid and bcc solid free energies, one lying on the low-density side and the other on the high-density side of it, coalesce into this single point. No susceptibility diverges and all free-energy-density curves remain strictly concave up.

It is now pertinent to ask whether the Hansen-Verlet freezing criterion is satisfied for both the low- and the high-density crystallization of the system. To this end, we have performed additional MC simulations at temperatures below $t_{\mathrm{u}}$ and in figure 10 we show structure factors at two such temperatures, $t=0.007$ and $t=0.005$, for increasing values of the density. It can be seen that the Hansen-Verlet criterion is indeed valid for both freezing transitions, a feature also observed for the re-entrant melting phenomenon in star polymer solutions [3,4].

It is of course also possible to calculate the exact free energies of the various candidate phases in a simulation by means, e.g., of the virial route in the fluid state and by employing the Frenkel-Ladd method $[3,4,8,45,46]$ in the solid state. However, the latter is very 

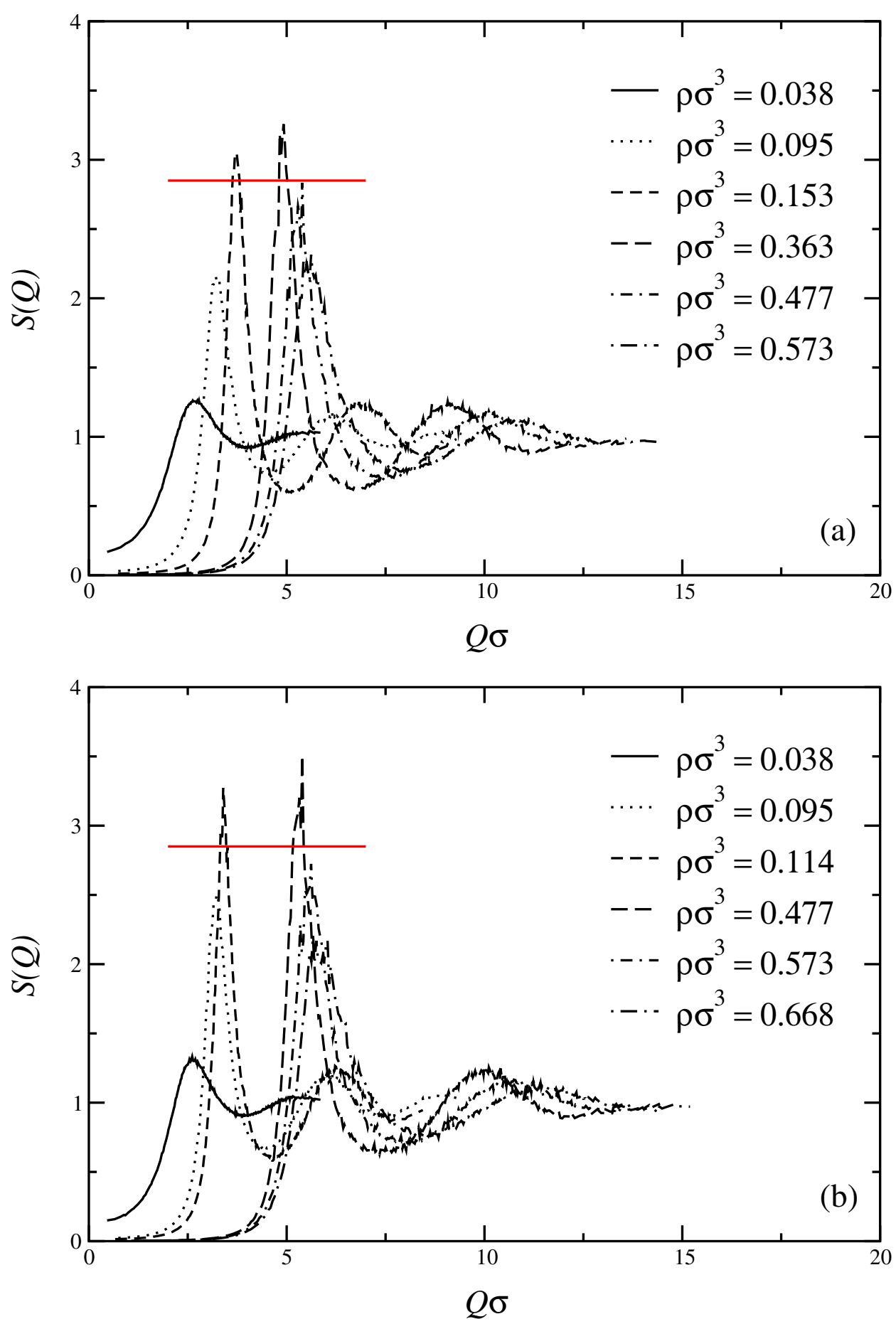

Figure 10. Structure factors $S(Q)$ for the GCM at (a) $t=0.007$ and (b) $t=0.005$ obtained from MC simulations. The densities are as indicated in the keys. The straight lines mark the HansenVerlet value 2.85. The corresponding structure factors in the regions $0.153 \leqslant \rho \sigma^{3} \leqslant 0.363$ for $t=0.007$ and $0.114 \lesssim \rho \sigma^{3} \lesssim 0.477$ for $t=0.005$ show Bragg peaks, indicating that the GCM has to be in the solid state for these densities. 
time consuming. The very good agreement between the phase boundaries obtained from the approximate theory presented here and the $\mathrm{MC}$ results regarding the height of the peak of $S(Q)$ and the spontaneous crystallization of the system in the simulation box give us confidence that the phase diagram of figure 9 is quantitatively correct.

\section{Summary and concluding remarks}

The Gaussian core model displays a whole range of unusual phenomena and properties: an anomalous dependence of the correlation functions on the density, a high-density 'ideal-gas limit', re-entrant melting and an upper freezing temperature. Many of these characteristics arise from the fact that the pairwise interaction does not diverge at zero separation, i.e., it is bounded. However, the topology of the phase diagram of the GCM has some striking similarities with that of star polymers [3,4], obtained by employing the diverging interaction given by equation (1). An upper freezing parameter ( $t$ in the GCM and $f$ in the star potential) appears in both, above which the systems remain fluid at all densities. Freezing and re-entrant melting occur also in both systems. Yet, the phase diagram of the GCM displays only two solid phases, whereas that of stars has a richness of exotic crystal structures [3]. The latter are caused by the crossover of the star potential from a logarithmic to a Yukawa form, a feature absent in the GCM.

To the best of our knowledge, the only other bounded interaction that has been studied by similar techniques to date is the penetrable spheres model (PSM) mentioned in section 2 [8-10]. The phenomenology and the associated phase diagram of the PSM are quite different from those of the GCM. These two bounded potentials have phase diagrams which do not look at all similar to one another. This comes as a striking difference from the relative insensitivity of the phase diagrams of unbounded interactions. In the PSM no re-entrant melting occurs and the system seems to freeze at all temperatures into increasingly clustered solids $[9,10]$. The reason for this difference is, evidently, that in the PSM, the particles can build clusters. In the GCM, this mechanism is not present; the interaction varies rapidly enough with distance, so multiple occupancies are penalized. Therefore, the boundedness of the interaction brings about a new factor to be considered: multiple occupancies, which are prohibited from the very beginning for diverging interactions, have to always be taken into account whenever one deals with bounded ones.

An important conclusion which appears to be valid for a large class of bounded potentials has been nevertheless drawn, and it is that of the mean-field ideal-gas behaviour of all such systems at high densities. The PSM also has a mean-field behaviour, although the discontinuity of the PSM pair potential at $r=\sigma$ forces its radial distribution function to have a jump at the same position at all densities and hence an ideal-gas limit can never be attained in this model. This is a feature complementary but not identical to the clustering property of the system. On the other hand, one can easily construct bounded pair interactions depending on some parameter, so that the sharpness of the decay of the PSM from a finite value to zero can be tuned. It will be very interesting to examine the structure and thermodynamics of such a family of systems as a function of the 'smoothing parameter' and establish the limits of the clustering and the ideal-gas behaviour at high densities. We plan to return to this problem in the future.

\section{Acknowledgments}

We thank Joachim Dzubiella, María José Fernaud, Professor J-P Hansen and Dr Ard Louis for helpful discussions, and Arben Jusufi for providing figure 1. This work was supported 
by the Österreichische Forschungsfond under Project Nos P11194-PHY and P13062-TPH. AL acknowledges financial support by the Deutsche Forschungsgemeinschaft within the Sonderforschungsbereich (SFB) 237.

\section{References}

[1] McConnell G A, Gast A P, Huang J S and Smith S D 1993 Phys. Rev. Lett. 712102

[2] Ilett S M, Orrock A, Poon W C K and Pusey P N 1995 Phys. Rev. E 511344

[3] Watzlawek M, Likos C N and Löwen H 1999 Phys. Rev. Lett. 825289

[4] Watzlawek M 2000 Phase Behaviour of Star Polymers (Aachen: Shaker)

[5] Likos C N, Löwen H, Watzlawek M, Abbas B, Jucknischke O, Allgaier J and Richter D 1998 Phys. Rev. Lett. 804450

[6] Jusufi A, Watzlawek M and Löwen H 1999 Macromolecules 324470

[7] Frenkel D 1999 Physica A 26326

[8] Likos C N, Watzlawek M and Löwen H 1998 Phys. Rev. E 583135

[9] Fernaud M J, Lomba E and Lee L L 2000 J. Chem. Phys. 112810

[10] Schmidt M 1999 J. Phys.: Condens. Matter 1110163

[11] Stillinger F H 1976 J. Chem. Phys. 653968

[12] Stillinger F H and Weber T A 1978 J. Chem. Phys. 683837

[13] Stillinger F H and Weber T A 1978 Phys. Rev. B 223790

[14] Stillinger F H 1979 J. Chem. Phys. 704067

[15] Stillinger F H 1979 Phys. Rev. B 20299

[16] Stillinger F H and Stillinger D K 1997 Physica A 244358

[17] Flory P J and Krigbaum W R 1950 J. Chem. Phys. 181086

[18] Olaj O F 1976 Makromol. Chem. 1773427

[19] Olaj O F, Lantschbauer W and Pelinka K H 1980 Macromolecules 13299

[20] Olaj O F and Lantschbauer W 1977 Ber. Bunsenges. Phys. Chem. 81985

[21] Grosberg A Y, Khalatur P G and Khokhlov A R 1982 Makromol. Chem. Rapid Commun. 3709

[22] Schäfer L and Baumgärtner A 1986 J. Physique 471431

[23] Krüger B, Schäfer L and Baumgärtner A 1989 J. Physique 503191

[24] Dautenhahn J and Hall C K 1994 Macromolecules 275933

[25] Louis A A, Finken R and Hansen J-P 1999 Europhys. Lett. 46741

[26] Alder B J, Hoover W G and Yound D A 1968 J. Chem. Phys. 493688

[27] Hoover W G and Ree F H 1968 J. Chem. Phys. 493609

[28] Haymet A D J and Oxtoby D 1986 J. Chem. Phys. 841769

[29] Hansen J-P and McDonald I R 1986 Theory of Simple Liquids 2nd edn (New York: Academic)

[30] Rogers F A and Young D A 1984 Phys. Rev. A 30999

[31] Verlet L 1980 Mol. Phys. 41183

[32] Lee L L and Shing K S 1989 J. Chem. Phys. 91477

[33] Lee L L 1992 J. Chem. Phys. 978606

[34] Lee L L 1995 J. Chem. Phys. 1039388

[35] Lee L L, Ghonasgi D and Lomba E 1996 J. Chem. Phys. 1048058

[36] Rosenfeld Y and Ashcroft N W 1979 Phys. Rev. A 201208

[37] Watzlawek M, Löwen H and Likos C N 1998 J. Phys.: Condens. Matter 108189

[38] Jucknischke O 1995 Untersuchung der Struktur von Sternpolymeren in Lösung mit Neutronenkleinwinkelstreuung PhD Thesis Westfälische Wilhelms-Universität Münster (in German)

[39] Hansen J-P and Verlet L 1969 Phys. Rev. 184151

[40] Hansen J-P and Schiff D 1973 Mol. Phys. 251281

[41] Evans R 1979 Adv. Phys. 28143

[42] Lebowitz J L and Percus J K 1966 Phys. Rev. 144251

[43] Ashcroft N W and Mermin N D 1976 Solid State Physics (Philadelphia, PA: Holt Saunders)

[44] Tejero C F, Daanoun A, Lekkerkerker H N W and Baus M 1995 Phys. Rev. E 51558

[45] Frenkel D and Smit B 1996 Understanding Molecular Simulation (San Diego, CA: Academic)

[46] Frenkel D 1986 Phys. Rev. Lett. 56858 